\title{
URBANISMO DE UN PUERTO “IMPERIAL” EN EL MEDITERRÁNEO. ESPECULACIÓN INMOBILIARIA Y RELACIONES tRANSNACIONALES EN LA EdAd MOdeRnA*
}

URBANISM OF A MEDITERRANEAN “IMPERIAL” PORT. REAL ESTATE SPECULATION AND TRANSNATIONAL RELATIONSHIPS DURING THE EARLY MODERN PERIOD

Francisco Zamora Rodriguez ${ }^{* *}$ fjzamorarodriguez@gmail.com

RESUMEN: El presente artículo analiza las consecuencias del crecimiento poblacional del puerto mediterráneo de Livorno y el surgimiento de nuevas oportunidades de negocio. El puerto de Livorno se presenta como un valioso observatorio a través del cual poner en diálogo asuntos como las políticas imperiales ibéricas, el urbanismo y las diferentes estrategias seguidas por los hombres de negocio durante la Edad Moderna.

PALABRAS-Chave: Alquiler, Livorno, Especulación.

ABSTRACT: This article analyses the consequences of the population growth in the Mediterranean port of Livorno and the emergence of new business opportunities. Livorno is presented as a valuable observatory when studying issues such as Iberian imperial policies, urbanism and economic strategies followed by traders during the Early Modern Period.

KEYWORDS: Rent, Livorno, Speculation.

\section{Introducción}

El crecimiento del puerto mediterráneo de Livorno durante todo el siglo XVII se concretó bajo los auspicios del gobierno de los Medici que supieron atraer a las redes del comercio internacional. La llegada de comerciantes de todas las naciones no se hizo esperar y Livorno se convirtió en puerto de redistribución enclavado en el Mediterráneo occidental pero con intereses puestos también en las mercancías americanas procedentes de los lucrativos mercados coloniales hispánicos y portugueses. En este marco se deben entender las actividades del cónsul español Andrés de Silva y de su homólogo portugués Pedro de Silva Enriques, pertenecientes a una misma familia de comerciantes. Por estos motivos, Livorno puede ser considerado en la práctica como un puerto global e "imperial", aunque en la teoría no perteneciera formalmente ni al entramado de la Monarquía Hispánica ni al de Portugal (ZAMORA RODRÍGUEZ, 2016). Como ha indicado recientemente Marcocci, a través de este perfil de comerciantes cosmopolitas, los Medici se aseguraron, por vía informal la

\footnotetext{
* Trabajo realizado en el marco del proyecto postdoctoral SFRH/BPD/89361/2012 financiado por la Fundação para a Ciência e a Tecnologia. Abreviaturas: ASLi: Archivo de Estado de Livorno; ASFi: Archivo de Estado de Florencia; NM: Notarile Moderno; Arr. Arroto; ASPi: Archivo de Estado de Pisa; AHN: Archivo Histórico Nacional de Madrid; BMo: Biblioteca Moreniana

${ }^{* *}$ Centro de História d'Aquém e d'Além-Mar (CHAM - FCSH/NOVA-UAç)
}

Hist. R., Goiânia, v. 21, n. 3, p. 29-46, set./dez. 2016 
participación en las rutas iberoamericanas en las que fueron incapaces de acceder de forma institucional (MARCOCCI, 2009).

\section{Crecimiento del puerto de Livorno en perspectiva comparativa}

En 1593, Fernando I de Medici promulgó las cláusulas de una segunda Livornina las cuales, más allá de ser utilizadas para ampliar los derechos y privilegios ya otorgados a los judíos en el año 1591 intentaba promocionar la llegada de comerciantes de otras naciones al puerto de Livorno. Se trataban de dos marcos jurídicos aplicados al recién creado puerto toscano de Livorno en el Mediterráneo occidental y que regulaban las condiciones que habrían de respetarse para el asentamiento de comunidades extranjeras. Concretamente, el texto de 1593 iba dirigido, según el prefacio, a los siguientes destinatarios (CASTIGNOLI; FRATTARELLI FISCHER, 1987):

A tutti uoi mercanti di qualsiuoglia natione, leuantini, e' ponentini, spagnioli, portoghesi, greci, todeschi, \& italiani, hebrei, turchi, e' mori, armenij, persiani, \& altri saluto (...) libero et amplissimo salvocondotto, facoltà e licentia che possiate venire, stare, trafficare, passare et abitare con le vostre famiglie o senza esse, partire tornare et negoziare nella nostra città di Pisa et terra di Livorno et anco stare per negoziare altrove per tutt'il nostro ducal dominio senza molestia alcuna reale o personale per tempo durante di 25 anni prossimi ${ }^{1}$.

El éxito de esta llamada fue rotundo. La llegada y asentamiento de comerciantes de diversa procedencia no se hizo esperar y, paulatinamente, el puerto de Livorno fue registrando un aumento de la población con un alto porcentaje de origen extranjero. Con respecto al puerto se decía en la época que "è uno dei più rinomati del Mediterraneo per la facilità del commercio e per la prodigiosa quantità di forestieri che da tutte le parti vi occorrono" ${ }^{2}$. La conformación de comunidades y sus diferentes estrategias de articulación y cohesión han sido objetos de estudios para la historiografía más reciente. Los estudios sobre las diferentes naciones asentadas en el puerto de Livorno y los límites de su reconocido cosmopolitismo han sido numerosos (PANESSA, 1998; BIAGI, 1978; ADDOBATTI; AGLIETTI, 2016).

Este proceso de crecimiento poblacional, gracias a la llegada de extranjeros y debido al establecimiento de un marco favorable de comercio, resultó análogo al que se produjo en

\footnotetext{
${ }^{1}$ Costituzione livornina de 1591, en Gutenberg E-book: shttp://www.gutenberg.org/files/19688/196888.txt>. Consultado en: 20 ago. 2016; Costituzione livornina de 1593, en Schoenberg Center for Electronic Text and Image: shttp://sceti.library.upenn.edu/sceti/ljs/pagelevel/index.cfm?ManID=ljs379\&Page=1>. Consultado en: 20 ago. 2016.

${ }^{2}$ BMo, Manoscritti Palagi, 385, ins.17, ff.4v-5r.
} 
puertos atlánticos como Cádiz donde, según revelan los datos del padrón de 1713, el 88,5\% de los hombres de negocios y comerciantes asentados en la plaza eran ya de origen extranjero (BUSTOS RODRÍGUEZ, 2005).

Para 1606, Ciano y Frattarelli cuantifican respectivamente la población de Livorno en 8.000 y 5.000 habitantes respectivamente. En menos de veinte años el puerto, que en 1590 contaba con 500 habitantes, habría multiplicado por diez su población. Por su parte, Mangio para 1642 cita 12.000 habitantes. Nuevamente Frattarelli, habla de 14.338 para 1662 y, finalmente, de 35.000 en 1750 (FRATTARELLI, 2003). Para 1668, el conde Galeazzo Gualdo Priorato estimaba la población de Livorno en cerca de 18.000 personas, añadiendo que se trataba del puerto más bello, cómodo y seguro de Italia ${ }^{3}$. El historiador de la época Gregorio Leti compartía la descripción de Galeazzo y afirmaba que en 1675 convivían en Livorno 20.000 personas y que la población crecía por días ${ }^{4}$. La diferencia cronológica entre estas dos obras, casi contempóraneas, es de solamente ocho años. Según la comparación de éstas, el incremento poblacional de Livorno habría sido de dos mil habitantes lo que puede darnos una idea de la evolución creciente del mismo. En términos demográficos, a mediados del siglo XVII, el puerto de Livorno se encontraba a la altura de los grandes puntos comerciales de la época y con mayoría de población extranjera. Livorno alcanzó en 1675 la población que Cádiz ya tenía a las alturas de 1638-1639, justo a las vísperas de su boom demográfico. Esta circunstancia nos da una idea aproximada en términos relativos de la población que el puerto mediterráneo tuvo en comparación con el puerto gaditano. En el arco cronológico de 16381675 Livorno ya había experimentado un notable incremento de habitantes cuyo inicio podríamos datarlo a comienzos del siglo. Cádiz, sin embargo, tuvo su expansión poblacional en torno al 1640 (que se prolongó hasta mediados del siglo XVIII). Sin duda, estos datos nos dan una visión comparativa del crecimiento de ambos puertos, uno enclavado en el Mediterráneo y otro en el Atlántico, concluyéndose que Cádiz, cuya potencialidad como fruto de su orientación a los negocios transatlánticos e imán para los extranjeros como fuente de riqueza americana, registró un crecimiento de una intensidad mayor en relación al de Livorno.

\footnotetext{
${ }^{3}$ GALEAZZO GUALDO PRIORATO, Relatione della città di Fiorenza e del gran ducato di Toscana, sotto il reqnante gran duca Ferdinando II consacrata all'Altezza Serenissimadel'Arciduchessa Anna d'Austria con tutte le cose più degne, e curiose da sapersi, Colonia, 1668. Se trata de una descripción de la ciudad de Florencia y del Gran Ducado de Toscana, conservado en la Biblioteca Nacional de Madrid.

${ }^{4}$ LETI, G. L'Italia regnante overo nova descritione dello stato presente di tutti Prencipati e Republiche di Italia, Génova, 1675, Tomo II, p. 297.
} 
Este último, sin embargo, también se vio favorecido por los intercambios atlánticos e igualmente tuvo una participación e importancia en el entramado imperial hispánico y portugués. Es por eso que podemos establecer una vinculación entre la prosperidad del comercio atlántico articulado desde Cádiz y el progresivo empuje poblacional de Livorno. Como si se tratase de dos vasos comunicantes, la interrelación comercial entre Atlántico y Mediterráneo gozó de una vitalidad vigente durante todo el siglo XVII y con un fluido contacto entre los principales enclaves italianos como Livorno y los puertos ibéricos como Lisboa, Sevilla y Cádiz.

Las dinámicas de atracción de capitales y redes comerciales puestas en marcha desde Livorno tampoco significaron una excepción en comparación con otros puertos de la península italiana. Estas estrategias fueron aplicadas tanto en el área mediterránea como en el Adriático y, gracias a las cuales, los puertos italianos jugaron un papel muy activo en la vehiculación del comercio atlántico como puertos receptores y redistribuidores de las mercancías americanas por los puertos mediterráneos (incluidos los pertenecientes al Imperio otomano como Esmirna, Aleppo o Alejandría) y Europa del Norte. En este sentido, fueron fundamentales las redes supranacionales judías, súbditas y extranjeras de los estados italianos (FUSARO, 2007; ZAMORA RODRÍGUEZ, 2014). Con resultados muy diferentes, en Ancona, Trieste y Venecia también se establecieron una serie de medidas con vistas al acrecentamiento de la actividad portuaria $y$, en definitiva, del beneficio económico de los diferentes estados a los que pertenecían estos puertos (ARBEL, 1995; RAVID, 1999, 2001; DUBIN, 1999). El caso veneciano, por ejemplo, fue muy notorio por la adopción en su territorio de comerciantes griegos y eslavos ortodoxos además de musulmanes turcos y judíos. A todos éstos se añadieron en el XVI los protestantes del norte (BURKE, 1988). Estos enclaves protagonizaron incluso una disputa por atraer y consolidar a estas redes judías en sus espacios portuarios (ANTUNES; TRIVELLATO; HALEVI, 2014).

En resumen, la prosperidad del puerto de Livorno durante el siglo XVII y buena parte del XVIII fue producto del intervencionismo estatal y apoyo convencido del gobierno florentino para la activación económica y comercial de la zona y de la que se benefició toda la región toscana. A través de diferentes marcos legales de carácter ecuménico, se establecieron los fundamentos para el crecimiento poblacional y económico de Livorno y, consecuentemente, se favoreció el asentamiento de numerosas comunidades extranjeras que 
quisieron participar de las ventajas ofrecidas. De esta manera, el gobierno florentino consiguió que Livorno se convirtiera en un nuevo punto de apoyo comercial en el Mediterráneo del momento, cuyo éxito radicó en su actuación como puerto de tránsito, depósito y redistribución (FRATTARELLI, 1993). Todo este proyecto trajo paralelamente nuevos desafíos, problemas y oportunidades de negocio relacionados con la capacidad estructural del puerto para dar acogida a un crecimiento poblacional de grandes dimensiones. Estas circunstancias han dado lugar a nuevos e innovadores análisis de otros casos portuarios en los que se ha analizado la gestión y gobernabilidad de los mismos (POLÓNIA DA SILVA y RIVERA MEDINA, 2016).

\section{Crecimiento urbanístico y especulación inmobiliaria}

Livorno se convirtió en una pequeña ciudad en cuanto a extensión urbanística pero con una densidad poblacional muy alta. El crecimiento poblacional de la ciudad y puerto de Livorno desembocó en una serie de problemas (aunque también oportunidades) relacionados con la capacidad infraestructural de un puerto con pretensiones globales. Entre los grandes desafíos a los que se enfrentó el puerto se encontraba no solamente la acogida de toda esta nueva masa social, sino también la cobertura de las necesidades derivadas de la conservación y salvaguardia de las mercancías mientras esperaban, en busca de los mejores precios y margen de lucro, para ser vendidas o redistribuidas hacia otros destinos. Livorno, sin embargo, afrontó este marco de crecimiento mejor que otros puertos vecinos también con un comercio floreciente. En Génova, por ejemplo, no fue hasta el 1681 cuando se reguló la creación de una cantidad de almacenes suficientes para ejercer una competencia real con el puerto de Livorno (GRENDI, 1987). Como consecuencia, Livorno se presentaba como la mejor opción para el comercio de mercancías de gran volumen. Incluso los mismos comerciantes de grano genoveses fueron acusados de negociar preferentemente desde Livorno aprovechando su mejor organización y posibilidades estructurales (GRENDI, 1987).

Livorno resultaba una ciudad cara para vivir y los problemas para encontrar alojamiento se extendieron incluso a los propios funcionarios locales. Además, la progresiva y lucrativa privatización de los bienes inmobiliarios estatales fue aprovechada por los comerciantes que invirtieron en la compra de estos espacios para ponerlos posteriormente en alquiler. Este proceso ha sido objeto de estudio en aplicación a diferentes puertos durante los siglos XIII al XVI (VVAA, 1988). En cierto sentido, "la Livorno mercantile resta città d'affitto, 
in cui anche la proprietà ha significato speculativo" (CONFORTO, 1984). Quizás el caso más significativo y mejor conocido de especulación inmobiliaria durante la Edad Moderna fue el llevado a cabo en el seno de la Monarquía Hispánica por el duque de Lerma. El válido de Felipe III aprovechó el movimiento poblacional que provocó la mudanza de la Corte de Madrid a Valladolid y viceversa para comprar bienes inmobiliarios en uno y otro lugar con el objetivo de revenderlos o ponerlos en alquiler. En ambos casos se benefició simultáneamente de la bajada y subida de precios de los inmuebles debido a la atracción poblacional, esencialmente de gente pudiente, que generaba la Corte como espacio de promoción, dádivas y oportunidades.

Los alquileres en Livorno sirvieron incluso como instrumentos para el pago de deudas. Tal es el caso del alquiler que Domenico Piacentini hizo a los hermanos Borgi (a razón de 180 ducados al año durante dieciocho años y medio) de la casa que el primero tenía en la via Ferdinanda de tal forma que pudiera ajustar la deuda de 3.800 ducados que tenía con la firma comercial Borgi. En una segunda operación, los Borgi se aseguraban el cobro en líquido de la deuda cediendo estos derechos al judío Abraham Attias que, en el momento de la firma, desembolsaba, por adelantado, 2.015 ducados por quince años y medio que residiría en esa casa $^{5}$. Éste es solamente un ejemplo de la importancia que fue adquiriendo la mercantilización y fiscalización de los alquileres inmobiliarios.

Por otro lado, la propiedad de bienes inmuebles tenía igualmente una serie de efectos sociales. No sólo era necesaria para la obtención de la ciudadanía en el puerto, y a la que solamente podían acceder los católicos, sino que también fue requisito básico para ser gonfaloniere, cargo municipal que garantizaba cierto poder de decisión en los asuntos locales. Además, el progresivo interés por la compra de inmuebles fue parte de un proceso en el que participaron los comerciantes y burguesías locales a través de la inversión en tierras, como método para desvincularse parcialmente de los negocios mercantiles - aunque nunca abandonaron completamente estas actividades-. Como ha expresado Angiolini, era una actitud que encontraba apoyo ideológico y justificación cultural con la teoría de la época sobre la incompatibilidad entre la nobleza y el comercio (ANGIOLINI, 1985). Los orígenes de esta tipología de inversión pueden encontrarse en el progresivo abandono, al menos en cuanto

\footnotetext{
${ }^{5}$ ASFi, NM, Protocolli, 17690 (años 1656-1661), ff.80r-91r. 9/6/1659. Cessio luria. La cesión por 15 años
} comenzaba el 17/7/1659. 
respecta a la gestión directa, de los negocios comerciales y la búsqueda, por parte de la élite mercantil portuaria, de nuevos nichos de negocio que se alejaran del negocio relacionado con la práctica directa de la mercatura, sobre todo de aquella que no era la transatlántica y comercio de larga distancia en general.

Como resultado de este crecimiento del mercado de alquiler y de las intenciones del gobierno florentino por sacar partido de él, en el 1646 Fernando II impuso una tasa del 8\% sobre los alquileres de las casas. La imposición de la decima, en un puerto que crecía en habitantes y cuyas actividades comerciales requerían un uso cada vez mayor del almacenaje, fue una estrategia medicea para recabar dinero e incentivar, a su vez, un mercado inmobiliario, dominado, al igual que el comercio, por los extranjeros. Esta decima, sin embargo, no la pagaban aquellos propietarios que hacían uso de los inmuebles, sino los que utilizaban estas propiedades para ponerlas en alquiler a otros. Por lo tanto, el $8 \%$ gravaba sobre la cuota que percibían estos propietarios de manos de los inquilinos que ocupaban el inmueble. Los grandes duques pusieron en marcha todo un programa de recaudación de impuestos donde la decima era uno de sus principales instrumentos (WAQUET, 1990). En un principio, fue la orden toscana de caballeros de Santo Stefano la que poseía gran parte de las propiedades, las cuales, eran concedidas en alquiler a numerosos judíos sefarditas que se fueron asentando en la ciudad ${ }^{6}$. Este tipo de circunstancias revelaba, sin duda, la existencia de una serie de relaciones interculturales y transnacionales articuladas a través de los alquileres y del lucro que éstos suponían para los propietarios.

Los fondos de la Decima granducal conservados en el Archivio di Stato de Livorno, nacidos como parte del mecanismo para controlar y recaudar este impuesto, son los que nos permiten reconstruir el aspecto urbano de Livorno por su detallada descripción física de los inmuebles y situación espacial de los mismos. Estos documentos nos revelan la compra y venta de las propiedades y también hacen una descripción física del espacio. Sin embargo, no nos arrojan luz sobre la puesta en alquiler de estos bienes, quiénes fueron los inquilinos o qué

\footnotetext{
${ }^{6}$ ASPi, Santo Stefano, 4629. Casas de propiedad de la orden de San Esteban puestas en alquiler en el puerto de Livorno. Relación de pagos (1678-1692). Una gran cantidad de judíos sefarditas reconocidos de la plaza portuaria se encontraban habitando estas casas en régimen de alquiler; ASPi, Santo Stefano, 4630. Es un legajo de características similares al anterior ( $n$ - 4629). A veces viene también indicado el origen del inquilino, se cita a armenios por ejemplo, y sobre todo está repleto de judíos. Otras fuentes también nos indican el estado floreciente de este mercado de compra y posterior alquiler de bienes inmuebles: ASFi, MP, 2280, 1/6/1685, "Registro di Beni Stabili e rendita di essi posti nella città di Livorno a cura del Proveditore di detta città suo porto e dogana”.
} 
precio pagaron por ellos. Para esto último hay que acudir a las cuentas particulares de los comerciantes y a los contratos de alquiler firmados con diferentes personas en el puerto, casi todos a través de los protocolos notariales.

El negocio especulativo de los alquileres también tuvo un efecto sobre los precios de los productos que se vendieron en Livorno ya que hicieron incrementar ostensiblemente el precio de venta por los elevados gastos ocasionados por el alquiler de almacenes. Así quedaba expresado también por un manuscrito de la época donde se afirmaba que cada negociante tenía sus almacenes más o menos en función de sus negocios y que la pigione (alquiler) era muy alta siendo ello el principal motivo del elevado precio de los productos ${ }^{7}$.

Esta diversificación de la inversión orientada al campo inmobiliario estaba también relacionada con la necesidad de cristalizar el éxito obtenido en los negocios y obtener rentas fijas. Este proceso no fue excepcional en Livorno. En el puerto gaditano, puerto fundamental en el funcionamiento del entramado imperial hispánico, se registró un proceso análogo que situó a la familia comerciante flamenca de los Colarte a la cabeza en cuanto a la inversión inmobiliaria y posterior alquiler de estos espacios (BUSTOS RODRÍGUEZ, 1991). Para la colonia británica en Cádiz también se ha evidenciado la inversión en bienes inmuebles. Fueron ilustrativos los casos de Diego Ricardo de Sussex o Guillermo Tirrick de Limerick (GARCíA FERNÁNDEZ, 2005). Por otro lado, en Milán, puerto seco fundamental para los negocios imperiales y la articulación del espacio italiano e internacional, también se han documentado estas actividades inmobiliarias. En esta ocasión fueron los Clerici, también comerciantes, los que dedicaron gran parte de su patrimonio a la compra y posterior puesta en alquiler de bienes inmuebles (MOCARELLI, 2003). De la misma manera, Chauvard ha llevado a cabo un profundo estudio sobre la circulación y utilización de estos bienes inmuebles en Venecia, especialmente durante el siglo XVII (CHAUVARD, 2003, 2005). También la ciudad de Sevilla ha sido objeto de estudios recientes donde se han analizado con detalle la evolución del mercado inmobiliario (propiedad y alquileres) entre los siglos XVI y XVIII (CARMONA GARCÍA, 2015).

En el 1684 se redactó el catastro livornés de propiedades. Once años después no se correspondía a la situación inmobiliaria en Livorno debido a que ésta había sufrido un continuo y sensible aumento, especialmente en cuanto respecta al valor de estos bienes inmuebles: de

${ }^{7}$ BMo, Manoscritti Palagi, 385, ins.17 f.12v. 
6.280 escudos de estimación se pasó a 12.000 escudos (FRATTARELLI, 2003). Es decir, en la segunda mitad del XVII, Livorno se enfrentó a una notable revalorización de los bienes inmuebles existentes y a un aumento de las construcciones. Ambas circunstancias quedaban integradas en una dinámica especulativa de carácter inmobiliario. A petición de los propios comerciantes del puerto se llevó a cabo, entre finales del XVII e inicios del XVIII la ampliación del espacio construido concretado en la edificación del barrio de Venezia Nuova que se servía de los canales de las fortalezas para el transporte de las mercancías. Estos años estuvieron caracterizados por una legislación medicea dirigida al control de este crecimiento estructural y a la necesidad de recaudar impuestos a través de la fiscalización de las ventas y los alquileres. En diciembre de 1704 se decretó el bando sobre la "decimazione degli stabili" por el que los propietarios debían declarar sus bienes al magistrado dedicado a la decima. Por sendas leyes de febrero de 1691 y de mayo del 1694 se reguló la recaudación de la decima. Por esta última del 1694 Cosimo III ordenaba a los poseedores de bienes inmuebles a que hicieran una estimación de los bienes inmuebles adquiridos con el objetivo de ejercer sobre ellos una imposición.

Vivir en alquiler era lo más común durante la Edad Moderna. Por tanto, la demanda siempre existía y, en casos de crecimientos poblacionales, las oportunidades lucrativas se multiplicaban exponencialmente. Efectivamente, el alquiler formaba parte del mecanismo económico de los mercados y supuso la obtención de una renta en el medio urbano por parte de ciertos grupos sociales (CAPEL SÁEZ, 2013).

Ejemplos de familias que invirtieron en las compras de viviendas y almacenes en Livorno aparecen continuamente. Tanto el cónsul español en Livorno Andrés de Silva como el representante de la Companhia Geral do Comércio do Brasil, Pedro de Silva Enriques, tío del anterior, se dedicaron a la compra y posterior venta en alquiler de diferentes propiedades. Concretamente, las compras realizadas por Andrés de Silva se concentraron básicamente entre los años 1692 y 1714 conformando un periodo cronológico tardío con respecto a otros negocios familiares. En efecto, la única compra registrada antes de este arco de tiempo fue en el 1677 y consistió en su propia vivienda, situada en Via Ferdinanda, una de las arterias de la ciudad, y donde antes había estado durante dos años en régimen de alquiler. Su adquisición respondía al deseo de asentarse en el lugar al igual que sucedió con otros cónsules como el 
francés o el genovés ${ }^{8}$. Antes, sin embargo, a nombre de su compañía comercial se habían comprado las primeras propiedades de la familia y Andrés de Silva adquirió continuamente propiedades. La ausencia de todas en los registros de la decima puede justificarse por el hecho de que no los pusiera en alquiler, al menos formalmente, no gravando sobre ellos el impuesto ${ }^{9}$. Otro de los hermanos, Manuel de Silva, también se dedicó a la compra de varios inmuebles con el objetivo de ponerlos en alquiler ${ }^{10}$. Este último aprovechó las posibilidades que ofrecían los grandes duques a los mejores postores e inversores a través de las subastas públicas de bienes embargados a sus propietarios o por cumplimiento de voluntades en los testamentos ${ }^{11}$. Este tipo de ventas eran anunciadas por el funcionario sonando una tromba. Hasta el 1584 el Ufficio dell'Asta Pubblica dependió de la aduana pero a partir de entonces se dio el cargo junto al cargo de camarero de la aduana de Livorno como una concesión. Las ventas se realizaban al abierto, en la puerta de la aduana, posteriormente se situarían en las Loggie della Comunità. Lo que se vendía eran mercancías confiadas por comerciantes privados al concesionario de la tromba: secuestradas por acreedores, puestas a subasta pública por

\footnotetext{
${ }^{8}$ AHN, Estado, 5010(I), 23/2/1690, fe de la vivienda de Andrés de Silva situada en la Via Ferdinanda y tomada en alquiler de la compañía de la Misericordia desde el año 1675. Véase ASLi, Decima, 209, arr.1072, 3/6/1677 compra por valor de 17.3.18.9 (referente al 8\% de la compra). Compra por Andrés de Silva a la compañía de la Misericordia de la casa situada en Via Ferdinanda con una tienda para el uso como almacén y una casetilla. Confina con los bienes alquilados por su tío Pedro de Silva Enriques.

${ }^{9}$ ASFi, NM, Protocolli, 16281, años 1664-1666, ff.92v-93r. 19/12/1665, venta de la compañía Odoardo y Luis de Silva de un terreno situado en la nueva ampliación de Livorno; ASFi, NM, Protocolli, 21095, Años 1697-1701, ff.87r-88v. 16/9/1699, Andrés de Silva aparecía en este protocolo como propietario de un bien inmobiliario en Livorno (en un lugar llamado Marignano); AHN, Estado, 5012, 13/3/1712, carta anónima dirigida al gran duque: "Servira la presente per partecipare alle signorie (...) sopra il particolare del memoriale che fa il marchese Andrea de Silva con il quale domanda a SAR a volerli concedere il diritto dominio d'un podere posto in questo piano di Livorno sul fiume della Cigna, che di presente è allivellato agli eredi di Silva Mendes per il quale si obliga di pagare pezze 500 conforme ho veduto da detto memoriale inviatomi dalle signorie".

${ }^{10}$ ASFi, NM, Protocolli, 22225, años 1701-1703, ff.171r-172r. 3/10/1703. Promissio. Compra de Manuel de Silva por 2200 piezas de reales de a 8 de una viña con casa y otras pertenencias detrás de los Capuchino junto a dos porciones de tierra (instrumento público de 1/9/1700). Compra de un huerto por 1300 piezas de reales de a 8 (22/3/1700); ASFi, NM, Protocolli, 21096, Años 1701-1706, ff.108r-109v. 24/7/1704. Los representantes de la cofradía de Santa María del Sufragio de Livorno formalizan solicitud de licencia al gran duque para vender a Manuel de Silva una porción de tierra que contenía una viña.

${ }^{11} \mathrm{ASFi}$, NM, Protocolli, 22224, años 1699-1701, ff.108v-109r. 1/9/1700, compra de Manuel de Silva (de Valencia y domiciliado en Livorno) de dos terrenos con sus propiedades adyacentes por el precio de 2.200 piezas de reales de a 8; íbidem, f.162rv. 22/3/1700. Compra de un huerto a subasta pública siendo Manuel de Silva el mejor postor por el precio de 1.300 piezas de reales de a 8 .
} 
orden judicial, presas marítimas o empeños no rescatados ${ }^{12}$. Andrés de Silva también recurrió a esta forma de compras para acrecentar el número de sus propiedades inmobiliarias ${ }^{13}$.

Las inversiones inmobiliarias en Livorno se repitieron ilustrativamente para los casos de Antonio Borgi, primer cónsul de los españoles, la familia Cotolendi, cónsules franceses en Livorno, y para los Ginori, una de las familias más influyentes del puerto, cuyo exponente máximo Lorenzo Ginori, había ostentado el consulado en Lisboa y posteriormente había vuelto a Livorno como Provveditore de la aduana ${ }^{14}$. A éstos se unieron familias cuyo éxito en los negocios y patrimonio era indiscutible como los Sproni, Franceschi o Attias. Otras familias portuguesas relacionadas con los Silva como los Mendes y los Silva Enriques poseyeron también entradas derivadas de la puesta en alquiler de bienes inmobiliarios ${ }^{15}$.

Aparte de la casa en via Ferdinanda, considerada uno de los mejores lugares de Livorno y donde habitaba gran parte de la burguesía comercial de Livorno, la decima gravó sobre otras propiedades de Andrés de Silva que se pusieron en régimen de alquiler y sobre nuevas construcciones y reformas realizadas en las diferentes propiedades. Estas propiedades y obras realizadas por parte de Andrés de Silva registradas en la decima ofrecen un reflejo de la fuerte inversión del comerciante:

\footnotetext{
${ }^{12}$ Información sobre las ventas al Pubblico Incanto (subasta pública). Tipologías de las mercancías puestas a la venta y el procedimiento para llevarla a cabo en BMo, Manoscritti Palagi, 385, ins.17, ff.10v-12v.

${ }^{13} \mathrm{ASFi}$, NM, Protocolli, 22223, años 1696-1699, ff.35v-36v. 17/8/1697. Andrés de Silva se hizo en el 5/7/1697 por 100 reales de a 8 como mejor postor de las dos porciones de tierra puestas en subasta al público incanto que pertenecían a la Casa Pia de Caridad de la ciudad de Pisa.

${ }^{14}$ ASLi, Decima, 33, f.1189, Compras de inmuebles de la familia Gavi, cónsules de la nación genovesa en Livorno; véase ASLi, Decima, 32, f.651; ASLi, Decima, 32, compras hechas entre marzo de 1691 y agosto de 1713 por Luigi Cotolendi de propiedades valoradas para la decima en 63.0.9.1; Para las compras en los años 1694 y 1695 de Lorenzo Ginori, véase ASLi, Decima, 33, f.868, compras valoradas para la decima en 48.2.16; ASLi, Decima, 213, arr.2281: casa de nueva fabricación cerca de la Fortezza Nuova. Se trata de una casa grande de 3 plantas y varios almacenes; Íbidem, arr.2299: compra de un almacén situado encima de otro (el 16/3/1694) a Francesco Carlesi en el barrio de Venezia Nuova; Íbidem, arr.2300, compra de otro almacén a nivel de tierra a Odoardo Brassart (el 17/2/1694) en el foso del Porticciolo; íbidem, arr.2355, construcción de almacenes en la casa que fabricó de nuevo mencionada anteriormente; Decima, 218, arr.733, compra inmobiliaria de Francesco y Carlo Ginori en 1711; Decima, 222, arr.62, compra de dos almacenes por parte de Giovanni Francesco Ginori en 1723 a los Sarmiento.

${ }^{15} \mathrm{ASLi}$, Governatore e auditore, Atti spezzati, leg.2195, ins.746, ff.774 s.n. 14/1/1685. Se trata de una causa de María de Silva Mendes (administradora de los bienes de Francisco Mendes) a su favor por el impago de una cuota de 25 escudos de Antonio Lumachi por el alquiler de un inmueble propiedad de los Mendes; ASLi, Governatore, Atti civili, leg.386, ff.1424-1426v. 7/7/1689. Causa de Bernardo Brandi contra María de Silva Mendes declarando el pago de todas las cuotas del alquiler; ASLi, Decima, leg.32, arr.305, año 1660 Pedro de Silva Enriques debía 14.2.16 a la decima; íbidem, f.402. Estado de las cuentas de la decima de Pedro de Silva Enriques a día 18/8/1661.
} 


\begin{tabular}{|l|l|l|l|}
\hline Fecha & Descripción & Situación & Vendedor \\
\hline $20 / 5 / 1692$ & ler piso de casa (dos habitaciones) & $\begin{array}{l}\text { Detrás de la Iglesia de los } \\
\text { Griegos }\end{array}$ & $\begin{array}{l}\text { Cammilo Ulivi y Pietro } \\
\text { Bizarri }\end{array}$ \\
\hline $28 / 7 / 1693$ & Nueva fabricación: 2 habitaciones & $\begin{array}{l}\text { En la casa de via } \\
\text { Ferdinanda }\end{array}$ & ---- \\
\hline $19 / 12 / 1702$ & 4 almacenes & $\begin{array}{l}\text { Via S.Giovanni (vicolo de } \\
\text { Campani) }\end{array}$ & Francesco Bicci \\
\hline $28 / 8 / 1703$ & Nueva fabricación: 7 tiendas & Pescheria Vecchia & ---- \\
\hline $3 / 9 / 1711$ & Dos habitaciones & $\begin{array}{l}\text { Detrás de la Iglesia de los } \\
\text { Griegos }\end{array}$ & $\begin{array}{l}\text { Convento y padres de } \\
\text { S.Antonio }\end{array}$ \\
\hline $5 / 1 / 1714$ & Reformas y ampliación & Pescheria Vecchia & Battista \\
\hline $24 / 7 / 1714$ & Stanzino no 18 en el segundo palco & $\begin{array}{l}\text { Teatro Público de } \\
\text { Livorno }\end{array}$ & $\begin{array}{l}\text { Giovanni } \\
\text { Buonfigli }\end{array}$ \\
\hline
\end{tabular}

Fuente: Elaboración propia según diferentes protocolos ASFi, $N M^{16}$

Entre los inquilinos de las posesiones de Andrés de Silva hubo gente de diversa procedencia y no puede establecerse un patrón que indique alguna preferencia por parte del propietario, quien no desdeñó ninguna posibilidad a la hora de lucrarse con el alquiler del inmueble. Aunque la mayor parte de ellos fueron italianos procedentes de Pistoia, Florencia, Génova o de la propia Livorno, Andrés de Silva contrató alquileres puntualmente con ingleses y franceses. En el primer caso, a través de un contrato firmado en noviembre de 1714 se le alquilaba a un inglés toda la primera planta que quedaba encima de las tiendas de la Pescheria Vecchia con la claúsula de que no diera escándalos. Esta observación no se encuentra en los demás contratos y es muy probable que se incluyera debido al reciente final de la guerra de Sucesión y al hecho de que fuera protestante. Más interesante, sin embargo, resulta el segundo de los casos: el alquiler a franceses. Las estrechas relaciones y objetivos comunes de los gobiernos francés y español en el primer cuarto del XVIII explicaban el alquiler de espacios a franceses por parte del cónsul Silva. Más complicado resulta explicar el controvertido alquiler de la casa de Via Ferdinanda, donde había vivido durante su periodo inicial en el puerto, al que fuera cónsul francés Varlet de Gibertcourt en pleno contexto bélico de la guerra de los Nueve Años y cuya llegada al puerto se debió a la conclusión de la firma de la neutralidad en Livorno debido a la muerte de Francesco Cotolendi. Varlet de Gibertcourt empezó a ejercer como cónsul de los franceses aproximadamente desde finales del 1691.

\footnotetext{
${ }^{16}$ Por orden de aparición en el cuadro, las referencias documentales utilizadas han sido tomadas en su totalidad de ASLi: Decima, 211, arr.1817; Decima, 212, arr.2080; Decima, 214, arr.2725, dos de los almacenes estaban a nivel de suelo y los otros dos a palco. Con anterioridad, estos 4 almacenes fueron comprados el día 16/2/1698 por Lucrecia y Silvia Daddi como herederas del Bicci y por el precio de 1590 piezas de a 8 reales; Decima, 214, arr.2790; Decima, 217, arr.716; Decima, 219, arr.970, comprado por 400 piezas de reales de a 8; Decima, 219, arr.1020.
} 
Los fines lucrativos privados dentro de un contexto socioecónomico complejo quedaban, inexorablemente, por encima de las relaciones interestatales de enemistad. La circunstancia podría entenderse en una relación contractual establecida entre dos comerciantes de la plaza. En el caso tratado, sin embargo, el beneficio privado sobrepasaba incluso el carácter representativo del propio cónsul y el compromiso con su estado de origen para el que, supuestamente, prestaba su compromiso y servicios ${ }^{17}$.

Haciendo un recuento de los beneficios reportados al cónsul Silva por las siete tiendas de su propiedad en la Pescheria Vecchia podemos analizar el alcance de esta lucrativa actividad. La no aparición de la tienda $n \div 4$ y la ausencia de inquilinos para algunas de las tiendas en diferentes periodos de tiempo puede deberse a varios motivos entre los que debe destacarse el que estuvieran utilizadas por el propio cónsul para el almacenaje y venta de los productos derivados de sus negocios particulares ${ }^{18}$ :

Contratos de alquiler de siete de las tiendas propiedad del cónsul Silva

\begin{tabular}{|l|l|l|l|l|}
\hline Fecha & Inquilino & Duración & Precio & Tienda \\
\hline $1 / 8 / 1703$ & Lorenzo Chigi & $1 / 8 / 1703-1 / 8 / 1706$ & 48 & 1 y 2 \\
\hline $1 / 8 / 1703$ & Antonio Bartolotti & $1 / 8 / 1703-1 / 8 / 1704$ & 24 & 3 \\
\hline $15 / 9 / 1703$ & Elia Sales & $15 / 9 / 1703-15 / 9 / 1704$ & 20 & 7 \\
\hline $1 / 2 / 1705$ & Gio.Marianini & $1 / 2 / 1705-1 / 2 / 1718$ & 24 & 5 \\
\hline $1 / 7 / 1705$ & Antonio Anichini (florentino) & $1 / 7 / 1705-1 / 7 / 1708$ & 24 & 7 \\
\hline $12 / 7 / 1708$ & Los Ruffini & $12 / 7 / 1708-12 / 7 / 1711$ & 24 & 7 \\
\hline $15 / 9 / 1708$ & Domenico Checcacci & $15 / 9 / 1708-31 / 5 / 1713$ & 24 & 7 \\
\hline $1 / 5 / 1713$ & Bartolomeo Lazzerini & $30 / 4 / 1714-30 / 5 / 1715$ & 24 & 3 \\
\hline $15 / 5 / 1713$ & Francesco Carvelli & $1 / 6 / 1713-1 / 6 / 1716$ rescinde & 24 & 7 \\
& & por quiebra en $20 / 2 / 1716$ & & \\
\hline $31 / 5 / 1713$ & Francesco Carneri & Desde 31/5/1713 & 24 & 7 \\
\hline $1 / 10 / 1714$ & Lorenzo Chigi & $1 / 10 / 1714-1 / 10 / 1717$ & 96 & 1,2 y 3 \\
\hline $1 / 9 / 1716$ & Domenico Checcacci & $1 / 9 / 1716-1 / 9 / 1719$ & 24 & 7 \\
\hline $1 / 5 / 1717$ & Maria Vittoria Badara (genovesa) & $1 / 5 / 1717-1 / 11 / 1717$ & 2 al mes & Cualquiera \\
\hline $1 / 3 / 1718$ & Gio. Marianini & $1 / 3 / 1718-15 / 3 / 1722$ & 21 & 6 \\
\hline TOTAL & APROXIMADO (1703-1718) & & 1316 & \\
\hline
\end{tabular}

Fuente: AHN, Estado, 4889(I) ${ }^{19}$. Cantidades en reales de a 8.

Las propiedades anteriores a 1692, también puestas en alquiler, eran muchas más y entre ellas se encontraban diferentes huertos, las dos plantas de las ampliaciones hechas a la

\footnotetext{
${ }^{17} \mathrm{AHN}$, Estado, 4889(I), 15/2/1693. Contrato de alquiler entre Andrés de Silva y Varlet de Gibertcourt por el cual el segundo desembolsó 125 ducados florentinos por la casa en Via Ferdinanda para el periodo de tiempo entre $15 / 2 / 1693$ y $15 / 2 / 1694$.

${ }^{18}$ Elaboración propia a partir de AHN, Estado, 4889(I). Los precios vienen expresados en reales de a 8 y son anuales salvo única indicación expresa en la que el pago es mensual. Las tiendas estaban clasificadas por números que son los indicados en la tabla.

${ }^{19}$ En este mismo legajo se conservan también los contratos de alquileres estipulados por Odoardo de Silva, el sobrino de Andrés de Silva.
} 
Pescheria Vecchia y una casa con quince habitaciones a las afueras del puerto, situada en un lugar significativamente conocido como Borgo Silva. Por otro lado, una propiedad que no aparece en los registros de la decima pero que el cónsul había comprado en 1694 era un terreno en Coteto, a las afueras de Livorno, que parceló y reformó para su puesta en alquiler. Este terreno fue objeto de un litigio jurisdiccional entre los Silva y una familia de la nobleza florentina justo después de que fuera cedido varias veces en régimen de enfiteusis ${ }^{20}$. Un contrato privado del cónsul Silva con Pietro Giannoni y Bastiano Viviani, hijastro del anterior, para que, como campesinos, labraran y cuidaran de estas propiedades en Coteto evidencia las relaciones de dependencia de varias personas con el cónsul y la probabilidad de que la familia se reservara una parte del terreno para su propio usufructo ${ }^{21}$. En este sentido, otra de las modalidades de alquiler utilizadas por Andrés de Silva fue la conocida como enfiteusis, o lo que es lo mismo, una figura jurídica por la que se permitía a un propietario ceder vitaliciamente un bien para el usufructo mediante el pago de un canon y cuya duración podía incluir varias generaciones. De esta forma, Andrés de Silva se aseguraba una entrada fija de dinero. En el contrato se establecían diferentes claúsulas convenidas por las dos partes. Otro de los bienes, tampoco reflejado en los registros de la decima granducal, era una viña con seis habitaciones y una terraza que fue concedida en enfiteusis a Giovan Battista Fagiolini, sus hijos, nietos y bisnietos hombres y en su defecto a las mujeres legítimas de éstos, con las condiciones de que debían pagar 40 reales al año como canon, junto a un único pago inicial de $30^{22}$. En el caso de dejar dos cuotas impagadas, la propiedad pasaría nuevamente al control

\footnotetext{
${ }^{20} \mathrm{ASFi}, \mathrm{NM}$, Protocolli, 24731 (1716-1719), ff.42r-43v. 26/1/1717 (Ya aparecía como cónsul de la nación española y marqués el sobrino de Andrés de Silva) Contrato de enfiteusis de una propiedad del marqués Odoardo de Silva a Francesco de Sebastiano Orlandi. Describe la propiedad situada en el lugar denominado Coteto: viña de dos saccate de extensión con casa de 6 habitaciones con terraza y columnata. Desde el 1/2/1717 empezarían a pagar 40 piezas de reales de a 8 (20 cada seis meses); ASFi, NM, Protocolli, 24733 (1720-1724), ff.23r-26r. 26/4/1721 (inc) Divisio. En ejecución de la sentencia y decreto del magistrado supremo de Florencia de 16/7/1720 se procedía, a través de este documento público, a la división en dos partes del terreno denominado Coteto que Andrés de Silva compró a Francesca Maria Rossano, mujer de Marc'Antonio Borghesi, de Siena el día 3/2/1694. Es una propiedad grande que Andrés de Silva mejoró con la construcción de algunas estancias adyacentes. A Odoardo de Silva se le dio la parte en la que se encontraban todas las mejoras realizadas por su tío. Odoardo tenía que pagar 220 piezas para igualar las dos porciones.

${ }^{21}$ AHN, Estado, 4889(I). Contrato estipulado el 14/8/1716.

${ }^{22}$ La compra de esta propiedad por parte de Andrés de Silva, y en el que heredaba el contrato enfiteútico después reconfirmado, se estableció a través del siguiente protocolo: ASFi, NM, Protocolli, 22223, años 1696-1699, ff.134v-136v. 16/2/1698. Venta. Francesco de Pietro Bicci ciudadano pisano que dejó en enfiteusis el 29/3/1670 a los hermanos Danielli 4 almacenes en la ciudad de Livorno en vía S.Giovanni. Tras la muerte de Francesco Bicci y viéndose su testamento en el cual deja herederas a sus hijas deciden vender el dominio de estos 4 almacenes a Andrés de Silva por 1.590 piezas de a 8 reales y que era todavía dependiente de los Danielli; ASFi, NM, Protocolli, 22225, años 1701-1703, ff.160v-161v. 30/8/1703, contrato de enfiteusis. Andrés de Silva, con pleno dominio
} 
de Andrés de Silva. Además, el inquilino se comprometía a tener en buen estado las viñas y a no descuidarlas. Sin duda, las claúsulas venían a cubrir los dos grandes problemas que acuciaban a los propietarios de bienes inmuebles en Livorno: los impagos y los daños causados por el uso inapropiado del inmueble ${ }^{23}$.

En base a la documentación de los alquileres de diferentes inmuebles entre 1700 y 1720, sin contar las tiendas del cuadro anterior, ni el alquiler de las tierras de pastoreo del feudo de la Banditella situado en Orbitelo, es posible estimar los ingresos de Andrés de Silva en unos 3.800 reales en total durante este periodo de tiempo. Aproximadamente, en conjunto, este negocio habría reportado a Andrés de Silva alrededor de 250-300 reales anuales.

A pesar de la gran cantidad de posesiones inmobiliarias en las que el cónsul pudo instalar su vivienda habitual, éste empezó a vivir de alquiler a partir de mayo de 1689 abandonando su casa en Via Ferdinanda. Con la muerte de su hermano Luis decidió trasladarse e instalarse en la casa del hermano pagando el alquiler de esta casa. La situación del inmueble ofrecía una mejor visibilidad y comodidad para instalar tanto la casa consular como la sede de su compañía ${ }^{24}$. La mentalidad de la época muy condicionada por parámetros como el honor y el prestigio actuaron como condicionantes debido a que esta casa estaba situada en la plaza Grande, uno de los lugares más exclusivos del puerto. Las casas consulares tenían la identificación de sus gobiernos en las puertas de entrada, de tal manera que pudieran ser identificadas por los miembros de sus respectivas naciones. En este sentido, posicionar la

sobre una viña con casa de seis estancias y terraza (entre otras posesiones) confinante con bienes de la compañía de Santa Giulia (alquilados a Milanesi), y otros bienes de Andrés de Silva.

${ }^{23}$ ASLi, Governatore, Atticivili, leg.580, s.n. 30/4/1717, proceso de Andrés de Silva contra los herederos de los Danielli. Andrés de Silva testimoniaba que siendo propietario directo de cuatro almacenes en la ciudad de Livorno situados en via S.Giovanni, concedidos en alquiler a los Danielli, solicitaba a la madre de los Danielli la cobertura de los gastos que se derivasen por la reestructuración de dichos almacenes que, por descuido y abandono, se habían estropeado estructuralmente; AHN, Estado, 5038(II), Año 1716 Escrituras pertenecientes a la bancarrota de Giuliano Camici. El inventario fue realizado por Andrés de Silva como representante de todos los acreedores. Entre los débitos contraídos estaba el alquiler de un almacén impagado a los Silva durante 5 meses (20 reales de a 8).

${ }^{24}$ AHN, Estado, 4889(I), 24/12/1688. Livorno. Stefano Cardi, testamentario de los hijos y herederos del caballero Giuseppe d'Angiolo de Livorno reconcede por otros 4 años el alquiler que Luis de Silva tenía de una casa propiedad del fallecido en Piazza Grande delle Armi. 220 escudos pagaba Andrés de Silva por esta casa; $12 / 12 / 1689$, Stefano Cardi como testamentario de los hijos y herederos del caballero Giuseppe d'Angiolo de Livorno concede en alquiler a Andrés de Silva (actuando en nombre de la razón Duarte, Luis y Andrés de Silva) una casa en Piazza Grande por 260 de a 8 reales anuales durante cuatro años desde el 1/5/1689; 1/2/1703. Por un escrito privado, el caballero Ranieri Battistad'Angiolo di Bonificarci le desquita a Andrés de Silva la cantidad de 17.6.8 de los 577.16.7 de a 8 reales que este último paga anualmente al caballero por las tres casas que le alquila. 
sede consular española en Piazza Grande, sin duda un lugar más privilegiado que el del consulado francés, entonces en régimen de alquiler en la casa de Andrés de Silva en via Ferdinanda, situaba simbólicamente a los españoles en una posición de superioridad frente al poder galo. En efecto, este traslado podía ser entendido en clave de éxito profesional y prosperidad en los negocios. A los propietarios de los inmuebles de este centro neurálgico, entre los que se encontraban el propio gobierno granducal y los caballeros de Santo Stefano, no les interesaba su venta por las altas rentas que podían recabar con su puesta en alquiler a los comerciantes más reputados.

\section{Conclusiones}

El puerto de Livorno se presenta como un valioso observatorio a través del cual poner en diálogo asuntos como las políticas imperiales, el urbanismo y las estrategias seguidas por los hombres de negocio durante la Edad Moderna. Desde su promoción estatal a través de la publicación oficial de una serie de ventajas y facilidades, el puerto de Livorno gozó de unas condiciones favorables que anunciaban una pronta prosperidad económica $y$, consecuentemente, un crecimiento poblacional y una reordenación urbanística enmarcada en un proceso mayor de globalización e integración creciente de los mercados. En este sentido, fueron las redes internacionales del comercio las que contribuyeron progresivamente a situar Livorno entre uno de los nodos de redistribución de mercancías más importantes del momento y en el que se daban cita las principales rutas internacionales del comercio. Entre éstas, destacaba, obviamente, la ruta del comercio atlántico y sus coneciones con los puertos ibéricos como Cádiz, Sevilla y Lisboa.

El asentamiento de nuevas comunidades junto al floreciente comercio ayudaron a que otras oportunidades de lucro pudieran ser aprovechadas por los hombres de negocio y, sin duda, por la fiscalización de nuevas actividades por parte del estado mediceo. En efecto, la construcción de nuevas viviendas, los necesarios almacenes para conservar el cada vez mayor volumen de mercancías y la puesta en alquiler de todos estos espacios hicieron de la especulación inmobiliaria un negocio muy lucrativo y generador de nuevas rentas e impuestos.

El papel desarrollado por este entorno urbano portuario resultó fundamental para las dinámicas imperiales, en muchos casos relacionadas con dar salida hacia otros mercados a sus productos coloniales. No en vano, entre las competencias principales del cónsul español 
y del agente de la Companhia Geral do Brasil estaba el facilitar el comercio de sus respectivos estados. Desde una óptica transnacional se han verificado unas relaciones intrapersonales entre los propios agentes ibéricos pertenecientes a una misma familia y que, como hemos comprobado, compartieron negocios relacionados con el ámbito inmobiliario.

\section{REFERÊNCIAS BIBLIOGRÁFICAS}

ADDOBBATI, A.; AGLIETTI, M. (Coord.) La città delle nazioni. Livorno e limiti del cosmopolitismo. Pisa: University Press, 2016.

ANGIOLINI, F. Nobles et marchands dans I'talie moderne. In: ANGIOLINI, F.; ROCHE, D. Cultures et formations négociantes dans l'Europe moderne. París: 1985, p. 103.

ANTUNES, C. A. P.; TRIVELLATO, F.; HALEVI, L. (Ed.) Religion and Trade: Cross-Cultural Exchanges in World History, 1000-1900. Oxford, 2014.

ARBEL, B. Trading nations: Jews and Venetians in the Early Modern Eastern Mediterranean. Leiden: 1995.

BIAGI, M. G. I Consoli delle Nazioni a Livorno, en Actas del congreso Livorno e il Mediterraneo nell'eta medicea. 1978, p. 361-368.

BURKE, P. Republics of Merchants in Early Modern Europe. In: BAECHLER, J.; HALL, J. A.; MANN, M. (Ed.). Europe and the Rise of Capitalism. Nueva York: 1988, p. 220-233, p. 222.

BUSTOS RODRÍGUEZ, M. Burguesía de negocios y capitalismo en Cádiz: los Colarte (1650-1750). Cádiz: 1991.

BUSTOS RODRÍGUEZ, M. Cádiz en el sistema atlántico. La ciudad, sus comerciantes y la actividad mercantil (1650-1830). Cádiz: Universidad de Cádiz, 2005, p. 138.

CAPEL SÁEZ, H. La morfología de las ciudades. Agentes urbanos y mercado inmobiliario, tomo III. Barcelona: Ediciones del Serbal, 2013, p. 282.

CARMONA GARCÍA, Juan Ignacio. Mercado inmobiliario, población, realidad social. (Sevilla en los tiempos de la Edad Moderna). Sevilla: Universidad de Sevilla, 2015.

CASTIGNOLI, P.; FRATTARELLI FISCHER, L. Le “Livornine” del 1591 e 1593. Livorno: 1987.

CHAUVARD, J. F. Rendita, diritto e morale. Proprietari e inquilini in età moderna, Quaderni storici, v. 11, 2003, p. 305-323.

CHAUVARD, J. F. La Circulation des biens à Venise. Stratégies patrimoniales et marché immobilier (1600-1750). Roma: 2005.

CONFORTO, M. L.; FRATTARELLI FISCHER, L. Dalla Livorno dei Granduchi alla Livorno dei mercanti. Città e proprietà immobiliare fra '500 e '600. In: Bollettino Storico Pisano, LIII, 1984, p. 211-234.

DUBIN, L. C. The ports of Jews of Habsburg Trieste. Absolutist politics and Enlightenment culture. Stanford: 1999. 
FRATTARELLI FISCHER, L. Livorno 1676: la città e il porto franco, In: ANGIOLINI, F.; BECAGLI, V.; VERGA, M. (Coord.). La Toscana nell'età di Cosimo III. Florencia: 1993, p. 45-66.

FRATTARELLI FISCHER, L. Case e proprietari a Livorno nel Seicento. In: Quaderni Storici, 113/a.XXXVIII, n. 2, ago. 2003, p. 369.

FUSARO, M. Gli uomini d'affari stranieri in Italia, In: FRANCESCHI, F.; GOLDTHWAITE, R. A.; MÜELLER, R. C. II Rinascimento italiano e l'Europa, v. IV Commercio e cultura mercantile, Vicenza, 2007, p. 381383.

GARCÍA FERNÁNDEZ, M. N. Comunidad extranjera y puerto privilegiado. Los británicos en Cádiz en el siglo XVIII. Cádiz: 2005, p. 83-85.

GRENDI, E. La repubblica aristocratica dei genovesi: politica, carità e commercio fra Cinque e Seicento. Bolonia: 1987, p. 351-353.

MARCOCCI, G. Itinerari marrani. I portoghesi a Livorno nei secoli dell'età moderna. In: PROSPERI, A. (Coord.). Livorno 1606-1806, luogo di Incontro tra Popoli e Culture. Torino: 2009.

MOCARELLI, L. Ascesa sociale e investimenti immobiliari: la famiglia Clerici nella Milano del SeiSettecento. In: Quaderni Storici, 113/a.XXXVIII, n. 2, ago. 2003, p. 419-433.

PANESSA, G. Nazioni e Consolati in Livorno. 400 anni di storia. Livorno: 1998.

POLÓNIA DA SILVA, A.; RIVERA MEDINA, A. M. (Ed.) La gobernanza de los puertos atlánticos, siglos XIV$X X$. Políticas y estructuras portuarias. Casa de Velázquez, 2016.

RAVID, B. A tale of three cities and their raison d'état: Ancona, Venice, Livorno and the competition for Jewish merchants in the sixteenth century, Mediterranean Historical Review, 6.2, 1999, p. 138-162.

RAVID, B. The Venetian government and the Jews. In: DAVIS, R. C.; RAVID, B. (Ed.). The Jews of Early Modern Venice, Baltimore, 2001, p. 3-30.

VVAA. D'une ville à l'autre. Structures matérielles et organisation de l'espace dans les villes européennes (XIIle-XVle siècle), Roma, 1988.

WAQUET, J-C. Le Grand-Duché de Toscane sur les derniers Medicis. Essai sur le système des finances et la stabilitè des institutions dans les anciens états italianes, Escuela Francesa de Roma, 1990, p. 217256.

ZAMORA RODRÍGUEZ, F. Interest and Curiosity: American Products, Information, and Exotica in Tuscany. In: ARAM, B.; YUN, B. (Ed.). Global Goods and the Spanish Empire, 1492-1824, Circulation, Resistance and Diversity, Palgrave, 2014, p. 174-193.

ZAMORA RODRÍGUEZ, F. Livorno al crocevia degli imperi iberici. In: AGLIETTI, M.; ADDOBBATI, A. (Ed.). La città delle nazioni. Livorno e i limiti del cosmopolitismo. Pisa University Press, 2016, p. 249-264. 